Memorias del 1 Congreso Nacional de Investigación en Enseñanza de la Biología. VI Encuentro Nacional de Investigación en Enseñanza de la Biología y la Educación Ambiental. ISSN 2027 1034. P. P. 83-92.

\title{
JUEGO DE SIMULACIÓN COMO PROPUESTA DIDÁCTICA PARA ABORDAR EL CONCEPTO EVOLUCIÓN BIOLÓGICA.
}

\section{SIMULATION GAME AS DIDACTIC PROPOSAL TO APPROACHING THE BIOLOGICAL EVOLUTION CONCEPT.}

POR: Luis Carlos Javier Ramírez Olaya ${ }^{1}$

\section{Resumen}

El documento aborda los resultados de la propuesta didáctica aplicada después de identificar algunas de las explicaciones utilizadas con mayor frecuencia por los estudiantes en relación con el concepto evolución biológica. En este orden se identificaron los obstáculos al aprendizaje más frecuentes en la comprensión del concepto, con el fin de plantear una estrategia clara y efectiva que trata de superar los mismos, puesto que la comprensión de la evolución biológica resulta fundamental para acceder a otras formas de búsqueda y pensamiento. En otras palabras, para disponer de respuestas alternativas a preguntas fundamentales en torno al origen y procesos de formación de las especies, incluida la nuestra.

Palabras clave: Juego de simulación, evolución biológica, representaciones, obstáculos al aprendizaje, emociones.

\section{Abstract}

This document shows the results about a didactic proposal applied after identifying some explanations, frequently used by students, in relation to the biological evolution concept. According to this, the most frequent learning obstacles in the concept comprehension were identified, with the aim of establishing a clear and effective strategy to overcome these obstacles, given that the biological evolution comprehension is fundamental to access other forms of search and thought. In other words, it is important to provide alternative answers for fundamental

\footnotetext{
1 luicro@hotmail.com. Licenciado en Biología.Universidad pedagógica Nacional.
} 
Memorias del 1 Congreso Nacional de Investigación en Enseñanza de la Biología. VI Encuentro Nacional de Investigación en Enseñanza de la Biología y la Educación Ambiental. ISSN 2027 1034. P. P. 83- 92.

questions related to the species origin and formation process, included the human being.

Key words: Simulation game, biological evolution, representations, learning obstacles, emotions.

\section{Introducción}

"Los conceptos de la ciencia, en toda su riqueza y ambigüedad, pueden presentarse sin ningún compromiso, sin ninguna simplificación que suponga distorsión, en lenguaje accesible a cualquier persona inteligente." Stephen Jay Gould. (1991)

Se propuso un juego de simulación ${ }^{2}$ (EVOLUCIÓN - Donde la carrera es sobrevivir y la meta es la reproducción -) concebido para superar algunos de los obstáculos al aprendizaje del concepto científico de evolución biológica, que fueron identificados en estudiantes del ciclo conceptual nivel C del Instituto Alberto Merani, durante la práctica pedagógica realizada en el año 2009. El juego fue aplicado en el 2010 a 29 estudiantes del ciclo conceptual, como una sesión de las cinco planteadas dentro de la propuesta didáctica.

A través del juego se pretende introducir elementos teóricos que posibiliten la comprensión del concepto de evolución biológica, el cual encuentra todavía objeciones y malinterpretaciones producto usualmente de su desconocimiento o mala comprensión. Básicamente se trata "de comprender que cada fenómeno material tiene una causa material y que los fenómenos observados no pueden ser explicados por causas no materiales. En otros términos, se trata de no utilizar elementos mágicos para explicar lo que se observa." (Gagliardi, s. f.)

Es evidente y no parece excesivo afirmar que el rol que desempeña el profesor de ciencias naturales en el aula incluye usualmente el paso del pensamiento común al conocimiento construido por la ciencia, en consecuencia, las ideas previas pueden funcionar como obstáculos en la comprensión del concepto, y deben ser conocidos por el profesor, puesto que "cuando el individuo aprende, no llena jamás un vacío sino que substituye poco a poco representaciones (intuitivas) que

\footnotetext{
${ }^{2}$ Los "juegos de simulación no son más que modelos para explicar e ilustrar una serie de hechos, que en el caso de las ciencias son los distintos fenómenos naturales (Marx 1984)". (Barberá, y Sanjosé, 1990, p. 49)
} 
Memorias del 1 Congreso Nacional de Investigación en Enseñanza de la Biología.

VI Encuentro Nacional de Investigación en Enseñanza de la Biología y la Educación Ambiental. ISSN 2027 1034. P. P. 83- 92.

expresan la visión que los alumnos, e incluso los adultos, tienen del mundo que les rodea". (Giordan, 1987, p. 105)

\section{Metodología}

La investigación es de carácter cualitativo en donde "interesa lo que la gente dice, piensa, siente o hace; sus patrones culturales; el proceso y el significado de sus relaciones interpersonales y con el medio" (Lerma, 2004, p. 72), esto en un contexto educativo con unas características específicas. La población bajo estudio son los estudiantes del nivel $C$ del ciclo Conceptual del Instituto Alberto Meraní y la muestra son 29 alumnos del curso GAMMA, del cual las edades oscilan entre 9 y 12 años.

La técnica con la cual se aborda la problemática planteada es el análisis de contenido cualitativo, el cual "permite verificar la presencia de temas, de palabras o de conceptos en un contenido." (Gómez, 2000) Permitiendo plantear unidades con la información sistematizada del test diagnostico y los cuestionarios (la unidad de cuantificación corresponde a los conceptos relacionados con el cambio biológico). En el análisis de contenido en un modelo cerrado "las categorías están predeterminadas por un investigador desde el comienzo, por una teoría de la cual se quiere testar las predicciones" (Gómez, 2000), correspondiendo en este caso a los obstáculos al aprendizaje del concepto evolución biológica identificados en los estudiantes.

La investigación fue dividida cuatro etapas, en la primera se realizó la búsqueda de fuentes de información que aportaron soportes teóricos y antecedentes al planteamiento de la investigación, en la segunda se diseñaron y adaptaron los cuestionarios con los cuales se recolectó la información. En la tercera etapa se aplicaron el test diagnostico, los cuestionarios y el juego de simulación; los cuestionarios uno y dos fueron usados con el fin de conocer las representaciones antes del juego, y los cuestionarios cuatro y cinco fueron aplicados después de las actividades propuestas, esto con el propósito de verificar si se presentó algún cambio en las respuestas de los estudiantes. En la cuarta fase se sistematizaron las respuestas de la fase tres, y luego se plantearon unidades de información con el fin de cotejar éstas con las categorías (obstáculos al aprendizaje) que posibilitaron el análisis. 
Memorias del 1 Congreso Nacional de Investigación en Enseñanza de la Biología.

VI Encuentro Nacional de Investigación en Enseñanza de la Biología y la Educación Ambiental. ISSN 2027 1034. P. P. 83-92.

\section{Juego de simulación EVOLUCIÓN - Donde la carrera es sobrevivir y la meta es la reproducción -}

La educación debe ser alegría, puesto que "el entrelazamiento cotidiano entre razón y emoción constituye nuestro vivir humano, y no nos damos cuenta de que todo sistema racional tiene un fundamento emocional," (Maturana, 2001, p. 8) por tanto las explicaciones sobre hechos materiales que uno acepta tendrán como punto de partida ciertas emociones ${ }^{3}$. A causa de ello se plantea un juego de mesa que tiene como objetivo introducir elementos teóricos primordiales para el aprendizaje del concepto evolución biológica.

El juego tiene como protagonistas a siete especies de insectos, las cuales serán simuladas por los estudiantes;

En las simulaciones se intenta modelar parte de una réplica casi idéntica de los fenómenos de la realidad, pues se presenta un modelo o entorno dinámico y facilita su exploración (la observación) y modificación a los alumnos, de manera inductiva o deductiva mediante la manipulación. Así pueden descubrir los elementos del modelo, sus interrelaciones, tomar decisiones y adquirir experiencia directa delante de unas situaciones que frecuentemente resultarían difícilmente accesibles en la realidad (Castro, 2008, p. 233).

Si esto es así, los insectos serán un medio en la enseñanza de la evolución biológica. Además, se propone acelerar el tiempo para poder hacer evidente (en ejemplos concretos) por qué y cuándo ocurre la competencia por el recurso; pareja, alimento, lugar para vivir y enemigos naturales, la selección natural (eliminación y reproducción diferencial), y la mutación.

Junto a lo anterior se exhiben adaptaciones de los insectos, como lo son sus aparatos bucales, que son estructuras que han divergido desde el ancestro común del cual provienen, los cuales posibilitan explotar el recurso alimenticio de distintas formas. Así mismo, el juego exalta el concepto de biopoblación al involucrar varios individuos por especie (distintos niños son individuos de la misma especie) y varias especies. También se utiliza la coloración críptica, que posibilita que el jugador no sea depredado, y de este modo pueda continuar en competencia por la

3 "Desde el punto de vista biológico lo que connotamos cuando hablamos de emociones son disposiciones corporales dinámicas que definen los distintos dominios de acción en que nos movemos. Cuando uno cambia de emoción, cambia de dominio de acción" (Maturana, 2001, p. 8). 
Memorias del 1 Congreso Nacional de Investigación en Enseñanza de la Biología. VI Encuentro Nacional de Investigación en Enseñanza de la Biología y la Educación Ambiental. ISSN 2027 1034. P. p. 83- 92.

reproducción (aquí es importante la pregunta ¿Por qué tiene esa coloración y no otra?).

Examinemos brevemente los contenidos del juego: consta de las instrucciones, siete especies de insectos (una ficha por cada individuo de la especie), un tablero que representa el hábitat; el cual incluye las casillas: de la metamorfosis (Huevo, Larva o Ninfa, Pupa y Adulto), de los recursos alimenticios (Hojas, Excremento, Sangre y Otros insectos), pareja, cripsis, depredador, comodín (antecesor común), hábitat favorable (lanza de nuevo), casilla de reproducción. Además un dado que posibilita avanzar en el tablero (incorpora azar). También incluye un tablero adicional donde están las fichas que cogerán los jugadores según caigan en las casillas que le resulten favorables.

\section{Resultados}

Los obstáculos al aprendizaje más frecuentes en las unidades de información presentados de mayor a menor incidencia quedarían así: Necesidad (20.6\%), causación espontanea (18.39\%), Ortogénesis (16.09\%), Direccionalidad del cambio producido por el agente $(13.79 \%)$, La selección procede sobre la misma población sometida a cambios $(9.19 \%)$, Noción tipológica de especie $(5.74 \%)$, Mutante somático (5.74\%), Teleología (3.44\%), Evolución transformativa (3.44\%), No se diferencia agente mutagénico de agente selectivo (2.29\%), Antropocentrismo (1.14\%).

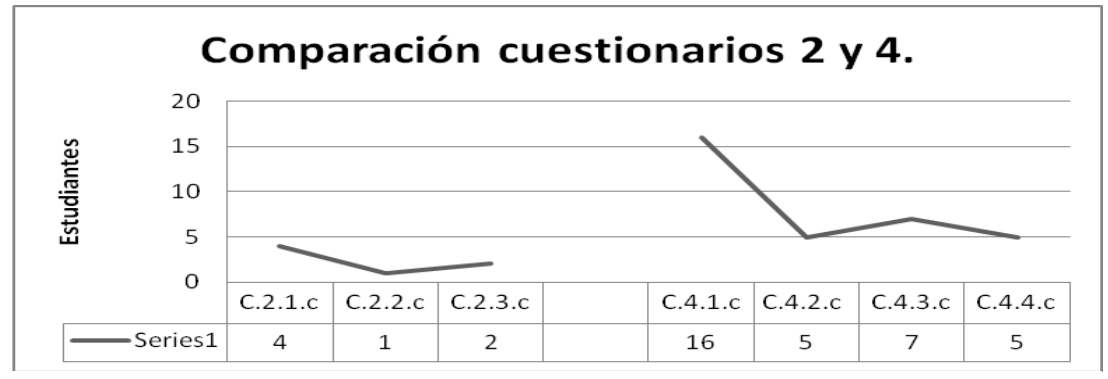

Gráfica 1Comparación de las respuestas acertadas de los cuestionarios 2 y 4.

En la gráfica 1 se presenta el número de estudiantes que seleccionaron las respuestas correctas de los cuestionarios 2 y 4 (de selección múltiple). El cuestionario 2 como fue aplicado antes del juego de simulación y el 4 después. Por tanto se evidencia un cambio positivo en las respuestas de algunos estudiantes después de las cinco sesiones planteadas. 
Memorias del 1 Congreso Nacional de Investigación en Enseñanza de la Biología.

VI Encuentro Nacional de Investigación en Enseñanza de la Biología y la Educación Ambiental. ISSN 2027 1034. P. p. 83- 92.

\section{Discusión}

Tras la revisión de las representaciones de los estudiantes y los obstáculos al aprendizaje del concepto científico de evolución biológica que se asientan en las mismas, sería insuficiente invocar una causa única a los problemas para el aprendizaje del concepto. La información circundante al estudiante aporta ideas previas utilizadas para la construcción de interpretaciones comunes de las cuales pueden resultar concepciones no apropiadas dentro del marco conceptual de la biología moderna.

Así mismo las explicaciones superficiales que dejan muchas preguntas por plantearse, y un nivel de escepticismo fluctuante que no le permite desarrollar un pensamiento crítico que duda de lo seguro, son posibles causas de la dificultad de la construcción del concepto y la persistencia de las ideas alternativas. Por lo anterior, es importante posibilitar un nivel tal de escepticismo que permita al estudiante no creer en todo sin plantearse preguntas que movilicen el aprendizaje de la ciencia (en el caso de la biología funcional ¿Cómo? y en la evolutiva ¿Por qué?), claro está que debe tener "un equilibrio exquisito entre dos necesidades conflictivas: el mayor escrutinio escéptico de todas las hipótesis que se le presentan, y al mismo tiempo una actitud muy abierta a las nuevas ideas" (Sagan, 1987), esto es necesario porque el estudiante puede preferir las respuestas que confirman su pensamiento común a las preguntas que lo contradicen.

Además de lo anterior, pueden existir obstáculos propios de la edad para la construcción del concepto, siendo esto enunciado de manera clara por Grau Sánchez (1993), quien después de una revisión de los estudios sobre las concepciones de la evolución biológica, expone "conjuntamente los resultados obtenidos para la variabilidad y la herencia por un lado y los referentes a adaptación y selección por otro".

Inicialmente los alumnos ( 7 a 13 años) no contemplan la variabilidad intraespecífica. Lógicamente no hay para ellos relación entre variabilidad, selección y adaptación (Deadman y Kelly 1978). En cualquier caso cuando explican la aparición de un nuevo carácter se expresan en términos lamarquistas, haciendo referencia al uso y falta de uso y a la necesidad (aunque sin mostrar un modelo de pensamiento excesivamente coherente) y acuden a una mezcla de sentido común y empiricismo (aquello que es plausible según su experiencia). Una interpretación mayoritaria contempla la influencia ambiental sobre las características heredables (Kargbo et al. 1980). (Grau, 1993) 
Memorias del 1 Congreso Nacional de Investigación en Enseñanza de la Biología.

VI Encuentro Nacional de Investigación en Enseñanza de la Biología y la Educación Ambiental. ISSN 2027 1034. P. P. 83- 92.

Lo anterior podría explicar por qué los obstáculos más frecuentes encontrados en la investigación fueron el de la necesidad, seguido por la causación espontánea, aunque debe tenerse en cuenta que en las respuestas a las preguntas del cuestionario tres, algunos estudiantes involucran la variabilidad intraespecífica (no en estas palabras) como un motivo por el cual no todas las bacterias mueren.

Habría que decir también que:

Para los alumnos más jóvenes, 11 a 13 años, la selección natural se relaciona con la extinción de algunas especies y la supervivencia de otras. Siempre se centra a nivel interespecífico. La adaptación sería una respuesta a un cambio ambiental o a problemas de supervivencia. En ambos casos se trata de una necesidad o un intento de perfeccionarse de los organismos, provocado por factores ambientales; existiría una voluntad, deseo o actividad consciente de reaccionar por parte de los organismos frente al medio. Muy pocos alumnos razonan en términos que se acepten actualmente como científicos, su modelo de razonamiento derivaría de una visión antropocéntrica del funcionamiento de la naturaleza (Deadman y Kelly 1978). (Grau, 1993)

Según los resultados obtenidos por Grau se podría aclarar porque el tercer obstáculo más frecuente en la investigación es el de la ortogénesis, seguido por la direccionalidad del cambio producido por el agente.

Es significativa la importancia que tiene el identificar las posibles causas de la dificultad en la construcción del concepto por parte de la muestra (29 estudiantes) de esta investigación, puesto que se producen datos de un contexto especifico, además de aportar una propuesta (el juego de simulación) de fácil manejo en el salón de clase para superar los problemas al aprendizaje del concepto. Pero es aun más trascendente la influencia que puede tener para el alumno y la población en general comprender el concepto de evolución biológica, puesto que además de posibilitar un acercamiento al pensamiento científico (observación y a partir de ella hacer inferencias por inducción y deducción) la "historia de la ética evolutiva es un tema que refleja tanto la teoría de la evolución como la ética. Debido a que explora los orígenes del hombre, la evolución extiende una invitación, y dado el estado de la ética, esa invitación ha sido atractiva," (Farber, 1994, p. 177) y quizás un enfoque diferente podrá ser valioso para descentrar el individuo, siendo esencial en la formación de nuevas ciudadanas y ciudadanos éticos frente a lo vivo y el ambiente. 
Memorias del 1 Congreso Nacional de Investigación en Enseñanza de la Biología.

VI Encuentro Nacional de Investigación en Enseñanza de la Biología y la Educación Ambiental. ISSN 2027 1034. P. P. 83- 92.

Por tanto, los sistemas vivos deben valorarse por su valor intrínseco; por su historia evolutiva y ascendencia común. Que les confiere un igual derecho de vivir en este planeta que compartimos. En este sentido Rozzi (1997) propone que:

Una nueva "ética de la tierra" es, a la vez, un deber moral y una actitud de prudencia en pro de la sobrevivencia humana. Desde el interior de la comunidad de seres vivos, debe despertar en los seres humanos un genuino amor, respeto, conciencia y obligación respecto a la totalidad de las manifestaciones de la vida. Desde el exterior, desde el análisis antropocéntrico, debemos considerar esta transformación ética, este cambio en nuestra relación con la naturaleza, como un paso indispensable para sobrevivir a nuestro propio impacto sobre el medio ambiente. (p. 10)

Lo anterior tendría como objetivo superar las influencias que reafirman el individualismo y la búsqueda del "progreso", las cuales son las raíces del "árbol" inmenso que sustenta nuestra palpitante crisis ambiental. $Y$ considerando que el cambio en el raciocinio moral puede tener efectos significativos en nuestra relación social, los argumentos podrán establecer nuevos principios (por ejemplo, igualdad racial y que los animales tengan derechos, para que no sean utilizados como cosas) y producir un cambio moral en la sociedad, viabilizando con esto que la próxima dimensión cultural construida por los colombianos sea acorde con las necesidades del siglo XXI.

Para terminar, habrá que tener en cuenta las posibilidades del juego (propuesto en esta investigación) en el destrozo del esencialismo biológico que impera en nuestra sociedad, por medio de lo que Mayr (2006) denomino "pensamiento poblacional", en el cual se identifica la variación entre individuos de una especie, viabilizando la introducción de conceptos imprescindibles en la comprensión de la evolución variativa.

Además, posibilita el desarrollo de valores como por ejemplo la solidaridad, la cooperación y el respeto por nombrar algunos. También influye en el acercamiento e interacción de los individuos en la clase, que aunque simple, no es fácil de lograrlo. Por lo anterior genera expectativas respecto al desarrollo de tres inteligencias de las ocho enunciadas por Howard Gardner en su teoría de las inteligencias múltiples. Estas son: la naturalista, intrapersonal y la interpersonal.

\section{Conclusiones}

Los resultados producto de la investigación realizada en el Instituto Alberto Merani, con 29 estudiantes (de 9 a 12 años) del ciclo conceptual nivel C, muestran que los 
Memorias del 1 Congreso Nacional de Investigación en Enseñanza de la Biología. VI Encuentro Nacional de Investigación en Enseñanza de la Biología y la Educación Ambiental. ISSN 2027 1034. P. P. 83-92.

obstáculos al aprendizaje más frecuentes presentes en las representaciones de los alumnos corresponden a: la necesidad, causación espontánea, ortogénesis y direccionalidad del cambio producida por el agente. Los cuales concuerdan con la revisión de las concepciones en el área de la evolución realizada por Grau (1993), en la que expone los resultados obtenidos teniendo en cuenta la edad de los alumnos. De 7 a 13 años concluye no contemplan la variabilidad intraespecífica y explican la aparición de un nuevo carácter haciendo referencia al uso y falta de uso, y a la necesidad. Además, es evidente una mezcla de sentido común y empiricismo en sus respuestas, y una interpretación regular es contemplar la influencia ambiental sobre las características heredables. Igualmente en estudiantes de 11 a 13 años la adaptación sería una respuesta a un cambio ambiental o a problemas de supervivencia, en donde se trata de un intento de perfeccionarse de los organismos, provocado por los factores ambientales; para lo cual existe una voluntad consciente de reaccionar.

El juego de simulación mostró ser eficaz en algunos estudiantes, puesto que se evidenció la introducción de conceptos esenciales para comprender el concepto evolución biológica. A pesar que unos conceptos fueron utilizados con anterioridad, después de las sesiones planteadas se emplearon adecuadamente. Estos son: variabilidad intraespecífica, selección natural (como reproducción y eliminación diferencial), herencia y adaptación.

\section{Agradecimientos.}

Al Instituto Alberto Merani por posibilitar el espacio y a los niños que participaron en la investigación. Al profesor Rodrigo Torres asesor del trabajo de grado. A Adriana Hurtado por la traducción del texto presentado en ingles.

\section{BIBLIOGRAFÍA.}

- Barberá, Ó. y Sanjosé, V. (1990). Juegos de simulación por ordenador: un útil para la enseñanza a todos los niveles. Enseñanza De Las Ciencias, 8 (I), p. 46-51

- Castro, Santiago. (2008). Juegos, Simulaciones y Simulación-Juego y los entornos multimediales en educación ¿mito o potencialidad? Revista de Investigación, № 65, p. $223-246$

- Farber, Paul. (1994) The Temptations of Evolutionary Ethics. Berkley: University of California Press.

- Gagliardi, Raúl. (s.f.). Los obstáculos al aprendizaje. Proyecto C.A.S. Recuperado el 10 Julio del 2010 de http://proyecto-cas.iespana.es/documen t/obstac2.htm 
Memorias del 1 Congreso Nacional de Investigación en Enseñanza de la Biología. VI Encuentro Nacional de Investigación en Enseñanza de la Biología y la Educación Ambiental. ISSN 2027 1034. P. P. 83-92.

- Giordan, A. (1987). Los conceptos de biología adquiridos en el proceso de aprendizaje. Enseñanza De Las Ciencias. 5 (2), p. 105 - 110

- Gómez, Miguel. Análisis de contenido cualitativo y cuantitativo: Definición, clasificación y metodología. Revista de Ciencias Humanas. No. 20. Recuperado el 16 de mayo del 2010 http://www.utp.edu.co/ chumanas/revistas/revistas/rev20/ gomez. htm

- Gould, Stephen Jay. (1991). La vida maravillosa. Barcelona: Editorial Critica.

- Grau, R. (1993). Revisión de las concepciones en el área de la Evolución. Enseñanza de las Ciencias, 11 (1), p. 87-89.

- Lerma, Héctor. (2004). Metodología de la investigación: propuesta, anteproyecto y proyecto. 3‥ ed. Bogotá: Ecoe Ediciones.

- Maturana, Humberto. (2001). Emociones y Lenguaje En Educación y Política. Dolmen Ensayo.

- Mayr, Ernst. (2006). Por qué es única la biología: consideraciones sobre la autonomía de una disciplina científica. Buenos Aires: Katz Editores.

- Rozzi, Ricardo. (1997). Hacia una superación de la dicotomía biocentrismo antropocentrismo. Revista Ambiente Y Desarrollo. Septiembre. Vol. XIII № 3

- Sagan, Carl. (1987). La carga del escepticismo. Recuperado el 10 Julio del 2010 de http://www.sindioses.org/escepticismo/cargaescepticismo.html 